\title{
Synergy between Adaptive Channel Coding and Media Access Control for Wireless ATM
}

\author{
Vincent K.N. Lau and Yu-Kwong Kwok \\ knlau@eee.hku.hk, ykwok@eee.hku.hk \\ Department of Electrical \& Electronic Engineering, \\ University of Hong Kong, Pokfulam Road, Hong Kong \\ Tel: (852)-28578483, Fax: (852)-25598738
}

25 February 1999

\begin{abstract}
In this paper, we propose mechanisms to exploit the synergy between the Media Access Control (MAC) layer and the physical layer for wireless ATM applications. For simplicity, the system considered consists of a single server and a single wireless ATM terminal. A number of virtual circuit connections (VC), with varying Quality of Service (QoS) requirements, are supported. We focus on two components of the wireless ATM system, namely the channel encoder and the ATM scheduler in the MAC layer. The channel encoder is responsible for protecting the ATM cells over the hostile radio channel while the ATM scheduler is responsible for allocating limited resources to the ATM cells for each virtual connection so as to meet the specified QoS requirements.

We consider two configurations, namely System-I with isolated adaptive channel encoder and ATM scheduler, and System-II with bi-directional information exchange. It is found that significant performance improvement on the Cell Loss Rate (CLR) and the Mean Cell Delay (MCD) could be achieved for systems exploiting the synergy.
\end{abstract}

\section{Introduction}

OSI 7 layers model are widely used in communication system design. The advantages are modularity and reusability. System designers usually focus on performance optimization within a single layer. However, the overall performance may not be optimal. In this paper, we try to exploit the synergy between adaptive ATM scheduler and variable rate adaptive channel encoder system.

For simplicity, the system consists of a single server and a single wireless ATM terminal as shown in figure 1.
A number of virtual circuit connections (VC), with varying QoS requirements, are supported. We focus on two components of the wireless ATM system, namely the channel encoder and the ATM scheduler in the MAC layer. The first component, namely the channel encoder, is responsible for protecting the ATM cells over the hostile radio channel. Variable rate adaptive channel encoder which adjusts the redundancy level based on the feedback channel state information is used ${ }^{1}$. The channel encoder subsystem is further discussed in section 2.

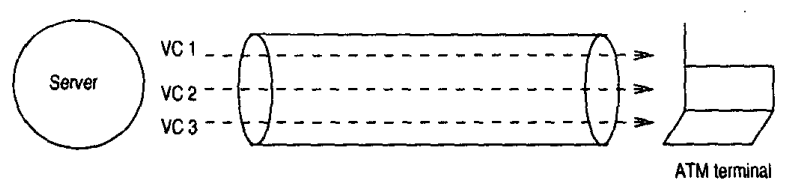

Figure 1: A wireless ATM terminal connected to an ATM server via a wireless link.

The second component, namely the ATM scheduler, is responsible for allocating resources to the ATM cells for each virtual connection according so as to satisfy the required QoS. A modified Earliest Deadline First (EDF) packet scheduler is employed as an illustration. The ATM scheduler subsystem is further discussed in section 3.

Synergy is achieved by exchanging information between the two subsystems. We considered a bidirectional information exchange mechanism which is further discussed in section 4.

Finally, we compare the performance of the two sys-

\footnotetext{
${ }^{1}$ When the channel state is good, the channel encoder reduces the amount of redundancy added to the ATM cells so as to boost the throughput. On the other hand, when the channel state is bad, more redundancy is added to maintain a target error rate.
} 
tems, namely System-I with isolated channel encoder and ATM scheduler, and System-II with bi-directional interaction mechanism. This is ellaborated in section 5 .

\section{Adaptive Channel Encoder}

For simplicity, we consider the single user case here. Physical frames are divided into $M$ ATM-slots. Each virtual circuit could occupy one or more slots depending on the instantaneous data rate requirement and available resources.

Figure 2 illustrates the datalink layer design employing a variable rate adaptive channel encoder to protect the ATM cells over the radio link. The motivation behind using the adaptive coding design is the significant improvement in the information throughput and the required signal-to-noise ratio (SNR) to achieve a certain bit error rate (BER) level $[1,2,3,4,5]$. Unlike any conventional error correction schemes, channel state information (CSI) which is estimated at the receiver is transmitted back to the transmitter side via a low-capacity feedback channel. Based on the CSI, the level of redundancy and the modulation constellation applied to the ATM cells is adjusted. Therefore, a variable instantaneous throughput is offered to the upper layers. In this paper, a 6-mode variable rate adaptive bit-interleaved trellis coded modulation scheme (ABICM) is employed [5]. As shown in figure 2, a number of encoding modes with normalized throughput varying from $1 / 2$ to 5 are available depending on the channel condition. Thresholds are set optimally [5] to maintain a target error level over a range of received signal-to-noise ratio (SNR).

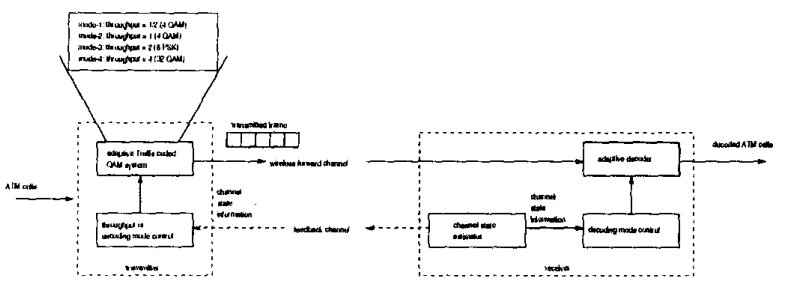

Figure 2: A conceptual view of the variable rate adaptive channel encoding system.

\section{$3 \quad$ ATM Cell Scheduler}

\subsection{Source Models}

The wireless ATM communication system considered in this paper is aimed to support various communication services for the user, such as data, voice, and video, each of which has its distinctive traffic characteristics and service requirements. Single data source is commonly modeled by a continuous Poisson arrival process or by a geometric interarrival process for the discrete case. The arrival process of packets from a voice stream is much more complicated than that of a data stream because there is a strong correlation among the arrivals. A Poisson process is commonly used for modeling the arrival process of new voice streams, the durations distribution of which can be modeled by an exponential distribution [6]. Within a single stream, there are alternating talkspurts and silent periods. For video streams, we assume the sources are VBR MPEG-2 encoded video data [7]. An MPEG-coded video stream can be characterized by three parameters: $q, n$, and $m$, where $n-1$ is the number of frames coded between successive I frames and $m-1$ is the number of B frames coded between successive $\mathrm{P}$ frames. One important observation of the MPEG coding method is that the data in an MPEG-code video stream are of unequal importance because the quality of a picture will not be significantly affected if packets from a $B$ frame are discarded.

\subsection{Scheduling Mechanism}

In the packet scheduling module, we use a modified earliest-deadline-first (EDF) algorithm [8] as an illustration for simplicity ${ }^{2}$. To better suit the user QoS requirements, which are assumed to be captured by two parameters: packet delay and packet dropping probability, we modify the traditional EDF scheduler as follows. The delay requirement induces deadlines for transmission of individual packets and is, therefore, handled by the inherent earliest-deadline-first criterion of an EDF scheduler. For the packet dropping aspect, our EDF scheduler is designed to be priority-based. As indicated above, different bit streams have different tolerance to loss. For example, data streams are highly intolerant to any loss, whereas voice or video streams can tolerate a certain degree of loss. Furthermore, even within a single video stream, some packets ( $B$ frame packets) are less important than others (I frame packets). Thus, we assume that after the packetization process, each ATM cell is associated with a priority metric, indicating importance of the packet. Thus, in addition to using the deadline misses as a criterion for discarding cells, the scheduler also checks whether the cells which are likely to miss its deadline is of a high priority or not.

\footnotetext{
${ }^{2}$ The concept of information exchange between the packet scheduler and channel encoder could be applied easily to other types of scheduler.
} 
Specifically, our modified EDF scheduler is a workahead scheduler in that it examines packets for scheduling within a time period $T$ (can be derived from the size of a frame of transmission) from the current time $t$. Suppose $d_{i j}$ is the deadline (induced from the delay requirement) of the $j$-th packet of the $i$-th stream. At time $t_{0}$, the modified EDF scheduler has to decide which packets to transmit in the next transmission frame, represented by the interval $\left(t_{0}, t_{0}+T\right)$. In a traditional EDF scheduler, the packets to be transmitted are those whose deadlines fall within the time interval. However, our modified EDF scheduler examines one time frame ahead (i.e., the interval $\left(t_{0}+T, t_{0}+2 T\right)$, to see if a straightforward earliest deadline scheduling in the current time frame will mandate discarding high priority packets subsequently. If so, the scheduler discards some of the low priority packets within the current time frame. Thus, this greedy look-ahead strategy can selectively degrade the performance of more loss-tolerant traffics. A schematic diagram of the scheduling mechanism is depicted in Figure 3.

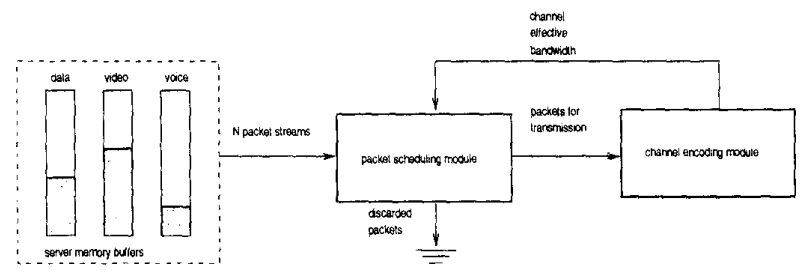

Figure 3: Overview of the packet scheduler.

\section{Synergistic Mechanisms}

Traditional system design focuses on performance optimization within a single layer. On one hand, coding specialists focus on designing error control coding in the data link layer and modulation schemes in the physical layer to transport raw information reliably without regard of the upper layers. On the other hand, protocol specialists focus on developing efficient resource allocation algorithms without interactions with the lower layers. These result in partially optimized systems. In this paper, we try to exploit the synergy by interaction between the packet scheduler and the channel encoder. Two levels of interaction mechanisms are proposed, namely the uni-directional information exchange and the bi-directional information exchange. They are illustrated in figure 4.

In the upward direction, the adaptive channel encoder communicates the offered instantaneous throughput to
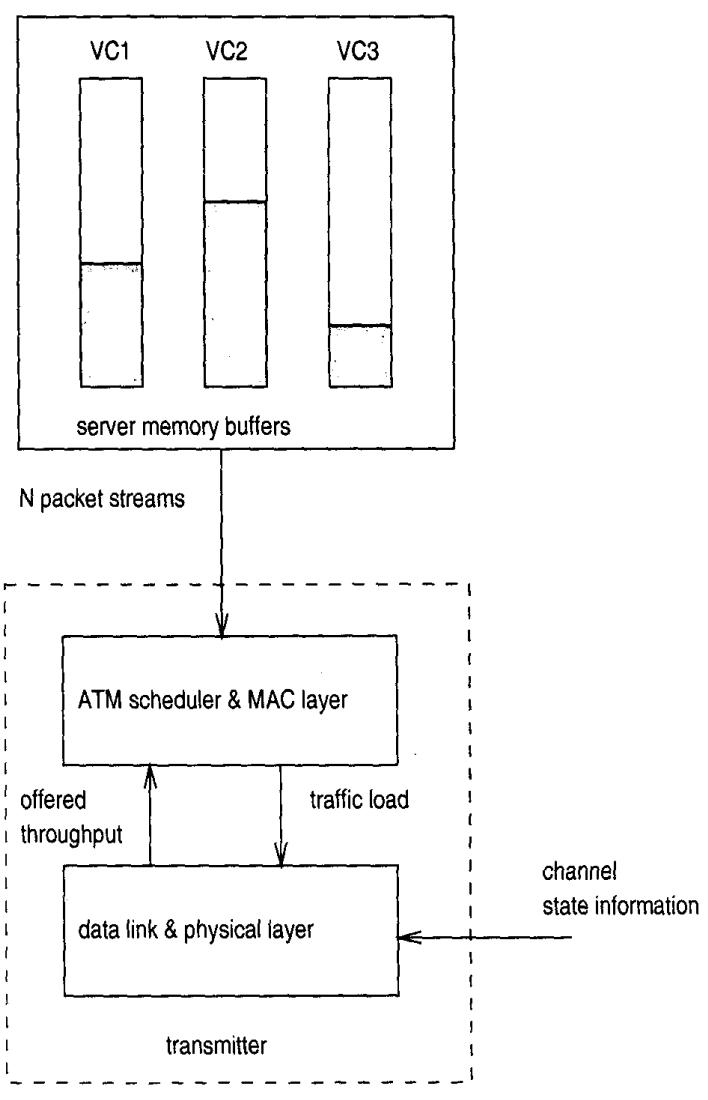

Figure 4: Illustration of the bi-directional interactions to exploit the synergy.

the ATM scheduler. The scheduler makes use of this extra information in the resource allocation (scheduling) decisions. For example, when the channel condition is good, a higher instantaneous throughput is offered to the ATM scheduler. Therefore, more ATM cells could be carried and the scheduler could dispatch more cells because of the temperal increase in resources.

In the downward direction, the scheduler communicates the instantaneous traffic loading information to the channel encoder. In the adaptive channel encoder, the offered throughput is directly related to the targeted bit-error rate $(B E R)^{3}$. Since the overall cell loss $(C L R(t))$ consists of two parts, namely the cell loss due to discarding at the transmitter $(C L R(d))$ and the cell loss due to errors at the receiver $(C L R(e))$. When the traffic loading is heavy, the ATM queues are heavily occupied and there is a high proportion of cells being

\footnotetext{
${ }^{3} \mathrm{~A}$ lower targeted BER level will result in a lower average throughput offered by the adaptive channel encoder.
} 


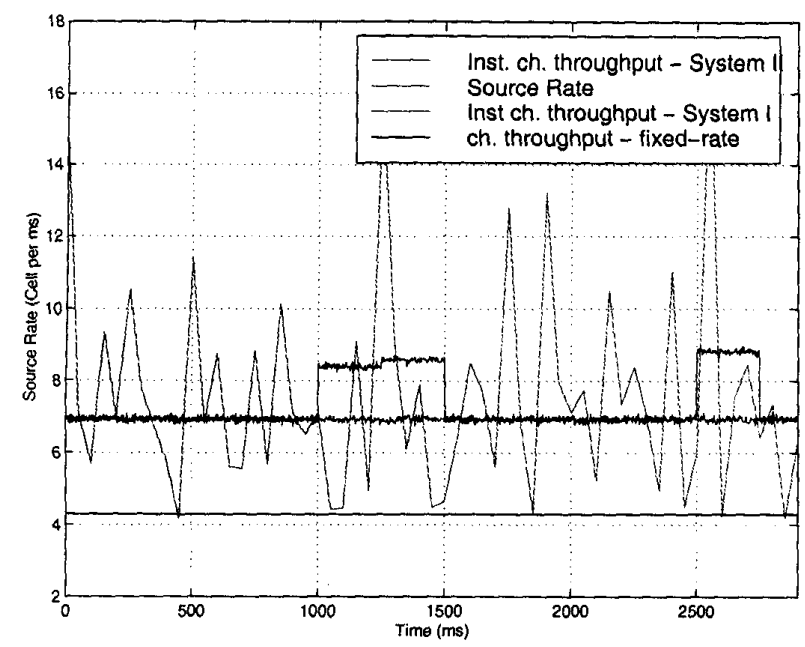

Figure 5: Burtiness of combined source and offered channel throughput.

discarded at the transmitter due to the scheduler's action. Therefore, it is no longer optimal to enforce a very low $C L R(e)$ at the channel encoder. A better setting at the adaptive channel encoder will be to raise the target BER level so as to increase the offered throughput. In this way, the $C L R(d)$ will be reduced. The scheduler communicates a traffic loading information to the channel encoder, requesting for an increase in the offered throughput. The channel encoder readjusts its thresholds to appropriately raise the target BER level to accomodate an increase in the offered throughput. Therefore, the downward interaction helps the channel encoder to determine an optimal target BER level relative to the traffic loading.

\section{Results and Conclusions}

We consider two configurations, namely System-I with isolated channel encoders and the scheduler, and System-II with bi-directional interaction mechanism. Simulations are performed and the results are summarized as follows. We focus on the Overall Cell Loss Rate (CLR) and the Mean Cell Delay (MCD). We have one video source (1.5Mbps), one TCP data source ( $384 \mathrm{kbps})$ and one UDP data source (64kbps) as background traffic. We also have $N$ voice sources (8kbps) as traffic loading parameter. Channel bandwidth is $833 \mathrm{kHz}$ with throughput of 2 bits per symbol as a baseline model. The burstiness of the combined source and the variation of the offered throuhgput are illustrated in figure 5.

\subsection{Isolated System}

With this setting, the adaptive channel encoder and the scheduler are connected with a buffer to smooth out the temporal variation in the offered throughput. There are significant improvements on the CLR and the MCD which is due to the increased average throughput offered by the ABICM relative to fixed rate code. This is illustrated in figure 6(a),(b). Average SNR $\left(E s / \eta_{0}\right)$ used is $18 \mathrm{~dB}$. The fixed rate coding could offered a throughput of 2 bits per modulation symbol with BER level of $10^{-4}$ as shown in the black line of figure 5 . For the adaptive encoder, the average offered throughput is 3 bits per symbol at the same conditions as shown in the pink line of figure 5. Therefore, the improvement in CLR and $M C D$ is due to the increased average throughput.

\subsection{Bi-Directional System}

We try to illustrate the synergy by looking at SystemII. Figure 6 illustrates the CLR and the MCD improvement. Note that there are improvements over the CLR and MCD for high congestion region. As traffic loading reduces, the performance of system-I and system-II merge. This is because at low traffic loading, the $C L R_{d}$ is essentially very low and the $C L R_{e}$ remains the same as the targeted error probability.

\section{Conclusion}

As a conclusion, this paper illustrates a mechanisms to exploit the synergy by interaction of upper and lower layers. We illustrate the concept by a single-user, multiple VCs system and consider the synergy between an ATM cell scheduler and an adaptive channel encoder. The results are very promising and this shed some light in the new direction of protocol design.

\section{Acknowledgement}

The authors would like to thank Prof. Victor Li for his stimulation on the idea of synergy.

\section{References}

[1] S. Alamouti and S. Kallel, "Adaptive Trellis-Coded Multiple-Phase-Shift Keying for Rayleigh Fading Channels," IEEE Trans. on Communs., vol. 42, pp. 2305-2314, June 1994. 


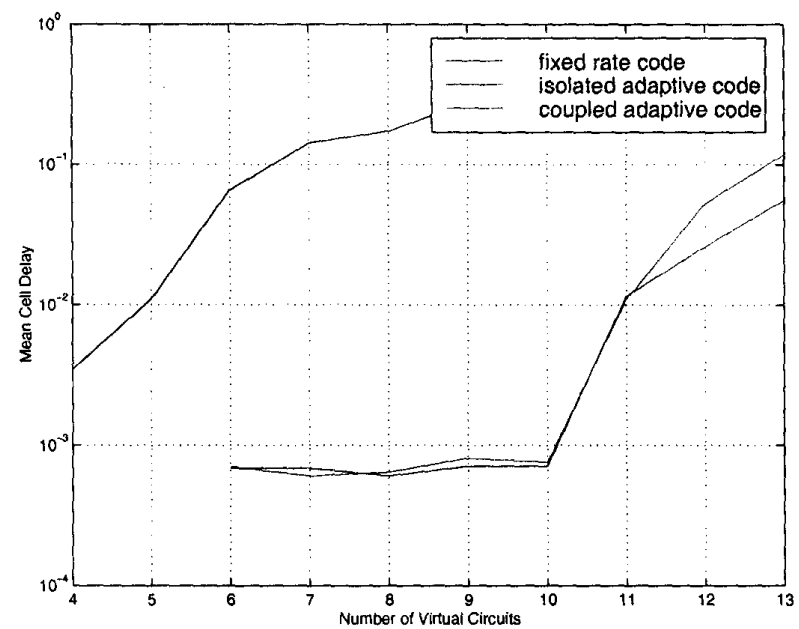

(a) CLR against $N$

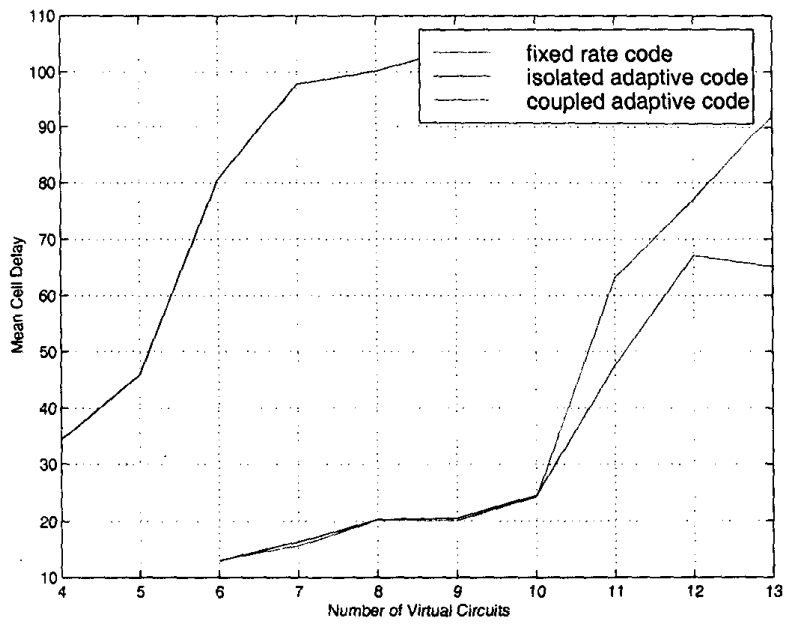

(b) MCD against $N$

Figure 6: Performance Comparison of fixed rate code, isolated adaptive code(System - I) and coupled adaptive code(System-II).
[2] W. Webb and R. Steele, "Variable Rate QAM for mobile radio," IEEE Trans. on Communs., pp. 2223-2230, July 1995.

[3] B.Vucetic, "An adaptive coding scheme for timevarying channels," IEEE Trans. on Communs., vol. 39, pp. 653-663, May 1991.

[4] K. Lau and S. Maric, "Adaptive Non-coherent Mary Orthogonal Modulation with RAKE Receiver for DS-CDMA," IEEE Trans. on Communs., April 1999.

[5] K. Lau, "On the design of Variable Rate Adaptive Bit-Interleaved Trellis Coded Modulation," Submitted to IEEE JSAC.

[6] J. Daigle and J. Langford, "Models for Analysis of Packet Voice Communications Systems," IEEE J. Selected Areas in Communications, vol. SAC-4, pp. 847-854, Sept 1986.

[7] T. Lakshman, A. Ortega, and A. Reibman, "VBR Video: Tradeoffs and Potentials," Proc. IEEE, vol. 86, pp. 952-973, May 1998.

[8] C. Liu and J. Layland, "Scheduling Algorithms for Multiprogramming in a Hard-Real-Time Environment," Journal of ACM, vol. 20, pp. 46-61, Jan 1973. 